\title{
BAIXO PESO AO NASCER NO BRASIL: REVISÃO SISTEMÁTICA DE ESTUDOS BASEADOS NO SISTEMA DE INFORMAÇÕES SOBRE NASCIDOS VIVOS
}

\author{
LOW BIRTH WEIGHT IN BRAZIL: A SYSTEMATIC REVIEW OF STUDIES \\ BASED ON THE LIVE BIRTHS INFORMATION SYSTEM
}

\author{
Dixis Figueroa Pedraza ${ }^{1 *}$ \\ 1dixisfigueroa@gmail.com \\ *Universidade Estadual da Paraíba
}

Data de entrada do artigo: 04/07/2013

Data de aceite do artigo: 03/12/2013

\section{RESUMO}

Introduçáo: $\mathrm{O}$ peso ao nascer é o mais importante fator de risco isolado a influenciar a sobrevivência infantil, devido à evidência da associaçáo entre o baixo peso ao nascer e a morbimortalidade infantil. Objetivo: Realizar uma revisão sistemática da literatura sobre o baixo peso ao nascer no Brasil, considerando os estudos que utilizaram a base de dados do sistema de informaçóes sobre nascidos vivos como fonte de informação. Métodos: Realizou-se busca ativa de estudos nas bases de dados MEDLINE, LILACS, e SciELO, utilizando-se a interseção dos termos "recém-nascido de baixo peso" e "sistemas de informaçáo" (o termo "Brasil" foi usado na busca no MEDLINE). Foram identificados 47 estudos nas bases de dados, dos quais 34 foram excluídos. Um total de 10 artigos foi incluído a partir das listas de referência dos 13 artigos inicialmente selecionados. Os 23 artigos incluídos foram caracterizados segundo a revista escolhida para publicação, local e ano de realização da pesquisa, metodologia e principais resultados. Resultados: Foram analisados 20 artigos transversais sobre o baixo peso ao nascer e três de tendência secular. A maioria dos estudos foi desenvolvida em âmbito municipal, mostrando uma ampla variação nos valores de prevalência, entre 5,6 e 10,6\%, não atingindo o patamar considerado como problema de saúde pública (15\%). Foram listadas 15 variáveis associadas ao baixo peso ao nascer, sendo as mais citadas: idade materna, número de consultas de pré-natal, sexo da criança, duração da gestação e escolaridade da mãe. Conclusão: Os resultados permitem definir prevalências aceitáveis de baixo peso ao nascer, assim como a influência da idade gestacional e de outros determinantes não biológicos, como a instrução materna e a adequação do pré-natal no peso ao nascer.

Palavras-chave: Recém-nascido de baixo peso; sistemas de informação; Brasil.

\section{ABSTRACT}

Introduction: The birth weight is the main isolated factor associated with infant's survival based on the fact that there is evidence of an association between low birth weight and infant morbidity and mortality. Objective: To report a systematic literature review about low birth weight in Brazil based on the Live Births Information System. Methods: A literature review was conducted at the MEDLINE, LILACS and SciELO electronics systems, using the intersection of "infant, low birth weight" and "information systems" terms (in MEDLINE the term "Brazil" was used). We identified 47 studies in the database, of which 34 were excluded. A total of 10 articles were included from reference lists of the 13 articles initially selected. The 23 articles included were characterized according to the journal, place and year of the research, methodology and main results. Results: Twenty of the analyzed articles were cross-sectional studies while three articles were about secular trends. Most of the studies were developed at municipal level. Prevalence of low birth weight ranged between 5.6 and $10.6 \%$, not reaching the level considered as a public health problem (15\%). 15 variables were listed, associated with the low birth weight, being maternal age, prenatal 
visits, child gender, gestational age and maternal schooling the most cited. Conclusion: The results allow setting acceptable low birth weight prevalence, as well the influence of the gestational age and other non-biological determinants such as maternal education and adequacy of prenatal on birth weight.

Keywords: Infant; low birth weight; information systems; Brazil.

\section{Introdução}

O peso ao nascer é o fator que individualmente mais repercute na saúde e sobrevida do infante, principalmente no primeiro mês de vida ${ }^{1}$, e influencia o crescimento e desenvolvimento da criança ${ }^{2}$. Além disso, é, provavelmente, também o fator que isoladamente tem maior importância na mortalidade neonatal, pós-neonatal e infantil, na morbidade durante a infância e no risco de várias doenças na idade adulta ${ }^{3}$. A categoria de peso entre 3.000 e 3.999 gramas é considerada a mais favorável, por representar um fator de proteção à mortalidade infantil ${ }^{1}$.

O baixo peso ao nascer (BPN) é definido pela Organização Mundial da Saúde (OMS) como todo nascido vivo com peso ao nascer inferior a 2.500 gramas $^{4}$. Os recém-nascidos de baixo peso apresentam prematuridade e/ou retardo do crescimento intrauterino (RCIU), sendo que os recém-nascidos pré-termos sáo associados mais frequentemente a fatores biológicos maternos e o RCIU, a fatores socioeconômicos. Não há, entretanto, uma linha divisória clara entre estes fatores, uma vez que fatores socioeconômicos podem ser mediados pelos fatores biológicos maternos e pelos cuidados pré-natais, assim como a presença de doenças pode afetar a situação socioeconômica. Ademais, as políticas sociais e a descentralização de serviços de saúde também são fatores relevantes, pois podem reduzir os efeitos da desigualdade da saúde 5 .

Estudos epidemiológicos mostram que a determinação da prematuridade, do RCIU e do BPN envolve um conjunto comum de fatores, entre os quais se destacam condições socioeconômicas precárias, baixo peso da mãe no início da gestação, tabagismo e estresse durante a gestação, falta ou deficiência da assistência pré-natal, antecedentes reprodutivos desfavoráveis e a ocorrência de gravidez múltipla ${ }^{6}$. A literatura também aborda a importância de outros fatores como consumo de café, ingestão de bebidas alcoólicas, peso da mãe durante a gestação, etnia, estatura da mãe, sexo do recém-nascido e morbidade materna durante a gravidez, principalmente por infecçôes perinatais, hipertensão arterial e disfunçôes uterinas ${ }^{2,3}$.

Deve-se considerar, ainda, que o estado nutricional da mãe, o tipo de dieta na gravidez e o tabagismo são importantes fatores que interferem no nascimento de recém-nascidos pequenos para a idade gestacional ${ }^{7}$. Considerando os determinantes que aparentemente são específicos do RCIU, destacam-se o consumo calórico insuficiente durante a gestação $0^{6}$, assim como outros fatores nutricionais, entre os quais, o déficit de estatura da mãe, a deficiência de micronutrientes (zinco, vitamina A e ácido fólico), o baixo Índice de Massa Corpórea (IMC) antes da gravidez e o baixo ganho de peso durante a gestação ${ }^{8}$. Por outro lado, as anormalidades placentárias e a incompetência do colo uterino são determinantes específicos da prematuridade ${ }^{6,7}$.

Em geral, nos países desenvolvidos as taxas de BPN são decorrentes, principalmente de partos prematuros, observados em dois terços dos nascimentos de crianças com BPN. Para os países em desenvolvimento, esta ocorrência se deve, na maioria das vezes, ao RCIU ${ }^{3}$. Entretanto, observam-se relatos do aumento do BPN em alguns países, e até mesmo em algumas cidades do Brasil, decorrentes do aumento de partos prematuros ${ }^{7}$.

Mundialmente, as taxas de BPN apresentam grande variação, com maiores taxas em países menos desenvolvidos, pois nesses países há associação de condiçóes socioeconômicas desfavoráveis ${ }^{3}$. Em países desenvolvidos da Europa, como Itália, França, Portugal, Suécia, Noruega, Irlanda e Espanha, o BPN varia entre 4,0 a 5,0\% \% $^{3,9}$. Nos Estados Unidos, essa taxa é de 7,6\%. As maiores proporçôes estáo na Índia $(33,0 \%)$ e em Bangladesh (50,0\%). Na América Latina e Caribe, a média é 9,0\% ${ }^{3}$. A Unicef cita Cuba, Costa Rica, Argentina e Venezuela como exemplos de países da região com prevalências de BPN entre 6,0 e 7,0\% no período de 1998 a $2002^{5}$.

No Brasil, em 2001, a média de BPN foi de 7,9\%, sendo a maior no Rio de Janeiro $(9,4 \%)$ e a menor em Roraima $(5,0 \%)^{3}$. A análise das taxas de BPN em 2005, considerando todas as macrorregiōes geográficas, destaca que as regióes Sudeste e Sul são as que possuem percentuais mais elevados $(9,0$ e 8,4\%). Já as regiōes Norte, Nordeste e Centro-oeste apresentam taxas de 6,8, 7,4 e $7,5 \%$, respectivamente. Os dados nacionais de 2005 indicam uma prevalência de $8,1 \%$, notando-se uma tendência estável quando comparados com o ano $2001^{10}$.

Muito do conhecimento existente sobre o BPN no Brasil tem sido possível devido à utilizaçáo do sistema de informações sobre nascidos vivos (Sinasc) como fonte de dados para publicaçôes científicas. Desta maneira, o objetivo do presente estudo foi realizar uma revisão sistemática da literatura sobre o baixo peso ao nascer no Brasil, considerando os estudos baseados no sistema de informaçôes sobre nascidos vivos. 


\section{Métodos}

Para a identificação dos artigos, realizou-se, em março de 2010, busca ativa de informaçóes nas bases de dados MEDLINE, LILACS e SciELO. A pesquisa bibliográfica considerou o tema central deste trabalho: estudos de baixo peso ao nascer baseados nas informaçôes do Sinasc. Com a finalidade de delimitar o objeto de estudo e o campo de investigaçáo para a realidade de estudo, optou-se por selecionar apenas produçóes na forma de artigos publicados em periódicos, considerando para o início do levantamento o ano de 1990. Esta opção está ligada ao fato da implantação do Sinasc ter ocorrido neste ano.

Os termos utilizados para a busca de artigos, realizada por dois revisores, foram: "recém-nascido de baixo peso" e "sistemas de informação", utilizando a interseção dos conjuntos. A busca foi realizada nos idiomas português e inglês. No caso da busca no MEDLINE o descritor "Brazil" também foi usado.

Para o cômputo do total de estudos identificados, foi verificada a duplicaçáo ou triplicação deles entre as bases de dados, sendo cada artigo contabilizado somente uma vez. A partir dos estudos identificados, considerando a leitura dos títulos e resumos pelos revisores, ou a leitura e análise criteriosa do texto na íntegra nos casos necessários, os estudos foram classificados como excluídos ou incluídos, considerando os critérios estabelecidos para esses fins:

- Critérios de inclusão: estudos sobre baixo peso ao nascer a partir de dados do Sinasc;

- Critérios de exclusão: estudos sobre baixo peso ao nascer com dados primários; estudos sobre avaliação da qualidade do Sinasc; estudos sobre mortalidade/ óbitos; estudos sobre prevalências de outros desfechos; estudos em outros países; artigos de revisão, livros ou teses.

Com o objetivo de localizar artigos que não tivessem sido encontrados nas bases de dados, a lista de referências dos estudos incluídos foi utilizada para a identificação de outros artigos, os quais foram avaliados respeitando-se os critérios de seleção.

Inicialmente foram identificados 47 estudos. Após análise dos títulos e resumos, ou do texto na íntegra nos casos necessários, foram excluídos 34 estudos, totalizando, portanto, a inclusão de 13 artigos que adequadamente preenchiam todos os critérios de seleção. A utilização das listas de referências bibliográficas dos artigos incluídos possibilitou a identificação de 10 artigos com os critérios pré-estabelecidos, resultando em um total de 23 artigos que fazem parte do presente estudo. O fluxo relacionado à inclusão e exclusão dos estudos encontra-se na tabela 1 . Os artigos incluídos foram caracterizados segundo revista escolhida para publicação, local e ano de realização da pesquisa, metodologia (populaçáo de estudo e critérios de inclusão adotados no estudo) e principais resultados (prevalências de BPN e variáveis associadas ao $\mathrm{BPN})$.

Tabela 1: Estudos excluídos e incluídos na revisão sistemática sobre o baixo peso ao nascer no Brasil, considerando os estudos baseados no Sistema de Informações sobre Nascidos Vivos (Sinasc)

\begin{tabular}{l|c}
\multicolumn{1}{c|}{ Etapas/Critérios de exclusão e inclusão } & $\begin{array}{c}\text { Número } \\
\text { de } \\
\text { artigos }\end{array}$ \\
\hline Estudos identificados & 14 \\
MEDLINE & 37 \\
LILACS & 04 \\
SciELO & 07 \\
Estudos em duplicata & 01 \\
Estudos em triplicata & 47 \\
Total de estudos identificados & \\
Estudos excluídos & \\
- estudos sobre baixo peso ao nascer com dados & 02 \\
primários & 04 \\
- estudos sobre avaliação da qualidade do Sinasc & 14 \\
- estudos sobre mortalidade/óbitos & 05 \\
- estudos sobre prevalências de outros desfechos & 01 \\
- estudos em outros países & 02 \\
- artigos de revisão & 06 \\
- livros ou tese & 34 \\
Total de estudos excluídos & 13 \\
Estudos incluídos & 10 \\
Estudos identificados e incluídos & 23 \\
segundo listas de referência & \\
Total de estudos incluídos & \\
\hline
\end{tabular}

A qualidade dos artigos foi avaliada através de checklist (lista de pontos) preparado pelos pesquisadores. O checklist consistiu de uma lista adaptada dos critérios de Downs \& Black $^{11}$, excluindo-se os itens relacionados apenas a estudos de intervençáo. Em relaçáo à construção do instrumento foi considerada a Declaração STROBE ${ }^{12}$ para a comunicação de estudos observacionais, e as seguintes diretrizes para a elaboração de listas de avaliação da qualidade de estudos observacionais ${ }^{13}$ : i) o instrumento deve ser de fácil utilização, ter um número pequeno de itens e ser do tipo checklist (evitar a medição em forma de escala); ii) o instrumento não deve deixar de considerar como foi realizada a seleção dos participantes, a medição das variáveis e o controle das variáveis de confusão, assim como o potencial do estudo/desenho para o controle dos vieses associados aos aspectos anteriores. 
Sendo assim, os artigos foram analisados com base na: (1) qualidade da descrição dos objetivos; (2) qualidade da descrição do desfecho de estudo; (3) qualidade da caracterização da amostra (descrição dos participantes e dos critérios de elegibilidade); (4) qualidade da descrição e discussáo dos principais fatores de confusáo; (5) qualidade da descrição das perdas de participantes; (6) qualidade da descrição dos principais resultados do estudo; (7) comprovaçáa da representatividade da amostra estudada em relação à população de estudo; (8) descrição do cálculo da amostra e do processo de amostragem; (9) acurácia dos instrumentos utilizados para medir o desfecho; (10) apropriação dos testes estatísticos às características das variáveis; (11) avaliação correta dos grupos de comparação (iguais períodos de seguimento para os estudos de coorte, iguais períodos de tempo entre a exposição e o desfecho para os estudos caso-controle); (12) adequação dos grupos de comparação (recrutados da mesma populaçáo e no mesmo período de tempo); (13) adequação do ajuste para os principais fatores de confusão ou apropriação dos testes estatísticos utilizados para o controle.

A qualidade do artigo correspondeu à soma do total de itens avaliados como positivos (escore de qualidade). Os escores de qualidade foram classificados nas categorias: i) qualidade alta (escore entre 9 e 13), qualidade média (escore entre 6 e 8), qualidade baixa (escore menor ou igual a 5).

Durante todas as etapas, os dois revisores trabalharam de forma independente. Os dados extraídos foram cruzados para a verificação da concordância. Os resultados discordantes foram resolvidos por consenso.

\section{Resultados}

Foram analisados um total de 23 artigos, sendo $20^{1,3,5,7,9,10,14-27}$ de cunho transversal e três ${ }^{6,28,29}$ de tendência secular. A tabela 2 mostra a distribuição quanto aos parâmetros de caracterização adotados.

Os periódicos Epidemiologia e Serviços de Saúde ${ }^{1,19,21}$ (anteriormente Revista Informe Epidemiológico do SUS), Cadernos Saúde Pública ${ }^{10,18,23}$ e Revista de Saúde Pública ${ }^{6,20,22}$ foram os que tiveram o maior número de artigos publicados, com três artigos publicados em cada um destes. No entanto, observa-se uma distribuiçáo uniforme quanto ao número de artigos publicados nos diferentes periódicos, o que leva a pensar sobre a importância dada ao tema e que não há um periódico preferido entre os pesquisadores para a divulgaçáo de estudos de BPN com a utilização de dados do Sinasc. Considerando os estudos de abrangência municipal, entre estes $11^{1,3,7,16,17,21,23-25,27,28}$ consideraram como população de estudo todas as crianças nascidas e residentes no município. Também cabe ressaltar que o principal critério de inclusão utilizado foi a gravidez única ${ }^{1,5,7,9,15,19,20,28}$.

A maioria dos estudos foi desenvolvida em âmbito municipal $(\mathrm{n}=18)^{1,3,7,9,15-28}$. Entretanto, uma pesquisa foi realizada considerando-se os dados de todo o território nacional, que abrangiam as cinco regióes do país ${ }^{10}$. O estado federativo onde houve maior frequência de estudos foi São Paulo $(n=6)^{3,6,17,19,20,26}$, sendo o município de Maringá o mais pesquisado $(n=3)^{9,14,15}$.

Os estudos realizados apresentam, como principais resultados, as taxas de prevalência de BPN e seus fatores associados, destacando-se, de um modo geral, a variabilidade nos resultados encontrados. A análise dos dados permite verificar a ocorrência do BPN, bem como estabelecer as desigualdades socioespaciais.

Os valores de prevalência variaram amplamente. Dos municípios estudados, as frequências menores de BPN foram encontradas nos municípios de Maringá (6,1\% no período de 1996 a 2002) $)^{9}$, Pariquera-Açu (6,6\% no ano 2002), Santo André $(6,8 \%$ no ano $2002)^{20}$ e Goiânia $(6,9 \% \text { no ano } 2000)^{16}$. Prevalências maiores que a média nacional $(8,1 \% \text { no ano } 2005)^{10}$ foram encontradas nos municípios de Belo Horizonte $(10,6 \% \text { no ano } 2001)^{23}$, Itararé $(10,4 \% \text { no ano } 2002)^{20}$, Passos (10,0\% no período de abril de 1995 a abril de 1996$)^{22}$, Feira de Santana $(9,7 \% \text { no ano } 1998)^{18}$, Campinas $(9,1 \% \text { no ano } 2001)^{3}$, São Paulo $(8,9 \%$ no ano 1998$)^{6}$, Blumenau $(8,7 \% \text { no ano } 1994)^{21}$, Rio de Janeiro $(8,7 \% \text { no ano } 2001)^{1}$ e Guaratinguetá $(8,2 \%$ no ano 1998$)^{19}$.

$\mathrm{O}$ estudo de abrangência nacional ${ }^{10}$ resultou em maiores prevalências de BPN, no ano 2005, para as regiôes Sudeste $(9,0 \%)$ e Sul $(8,4 \%)$, enquanto o Norte $(6,8 \%)$ e o Nordeste $(7,4 \%)$ apresentaram as menores taxas no indicador. $\mathrm{O}$ único estudo de abrangência estadual apontou prevalência baixa de BPN (5,96\%) para o estado de Goiás5.

Fazendo uma análise comparativa dos resultados obtidos, quatro aspectos importantes merecem ser destacados em relação à variação nas taxas de $\mathrm{BPN}$ : i) diferenças entre municípios de um mesmo estado (entre os municípios do Estado de São Paulo) $)^{3,17,19,20,26}$, ii) diferenças entre município e o Estado ao qual pertencem (entre o município de Goiânia ${ }^{16}$ e o Estado de Goiás no ano 2000), iii) diferenças entre dados hospitalares e o município ao qual pertencem (entre um hospital do município de Maringá ${ }^{14}$ e os dados de abrangência municipal) $)^{9,15}$, iv) diferenças na tendência ao longo do tempo sem poder vislumbrar alguma coerência ou lei geral de variaçẫo $0^{6,7,9,17,21,25,28,29}$.

Com relação à análise etiológica, foram listadas 15 variáveis associadas ao BPN, sendo as mais citadas: idade materna ${ }^{1,3,5-7,9,15,16,18-20}$, número de consultas 
Tabela 2: Características dos artigos sobre baixo peso ao nascer no Brasil com base nos dados do Sistema de Informações sobre nascidos vivos (Sinasc).

\begin{tabular}{|c|c|c|c|c|c|c|}
\hline \multirow[b]{2}{*}{ Autor, ano } & \multirow{2}{*}{$\begin{array}{l}\text { Revista de } \\
\text { publicaçáo }\end{array}$} & \multirow{2}{*}{$\begin{array}{l}\text { Local, ano do } \\
\text { estudo }\end{array}$} & \multicolumn{2}{|c|}{ Metodologia } & \multicolumn{2}{|c|}{ Principais resultados } \\
\hline & & & $\begin{array}{l}\text { População de } \\
\text { estudo }\end{array}$ & $\begin{array}{l}\text { Critérios de } \\
\text { inclusão }\end{array}$ & Prevalência de BPN & $\begin{array}{l}\text { Variáveis associadas ao } \\
\text { BPN }\end{array}$ \\
\hline \multicolumn{7}{|c|}{ Estudos transversais } \\
\hline $\begin{array}{l}\text { Barbas et al., } \\
2009^{1}\end{array}$ & $\begin{array}{l}\text { Epidemiol } \\
\text { Serv Saúde }\end{array}$ & $\begin{array}{l}\text { Rio de Janeiro } \\
(\mathrm{RJ}), 2001\end{array}$ & $\begin{array}{l}\text { Todas as crianças } \\
\text { nascidas e residen- } \\
\text { tes no município }\end{array}$ & $\begin{array}{l}\text { Gravidez única } \\
\text { Peso ao nascer } \\
<4.000 \mathrm{~g}\end{array}$ & $8,7 \%$ & $\begin{array}{l}\text { i) Análise univariada: } \\
\text { - Duração da gestação } \\
\text { - Idade materna } \\
\text { - Número de consultas de } \\
\text { pré-natal } \\
\text { - Escolaridade da mãe } \\
\text { - Sexo da criança } \\
\text { ii) Modelo com níveis } \\
\text { hierárquicos de } \\
\text { determinação: } \\
\text { - Duração da gestação } \\
\text { - Idade materna } \\
\text { - Sexo da criança }\end{array}$ \\
\hline $\begin{array}{l}\text { Silva, Pelloso, } \\
2009^{14}\end{array}$ & $\begin{array}{l}\text { Rev Esc } \\
\text { Enferm } \\
\text { USP }\end{array}$ & $\begin{array}{l}\text { Maringá (PR), } \\
2006\end{array}$ & $\begin{array}{l}\text { Todas as crianças } \\
\text { nascidas em um } \\
\text { hospital-escola do } \\
\text { município }\end{array}$ & - & $23,6 \%$ & $\begin{array}{l}\text { - Número de consultas de } \\
\text { pré-natal }\end{array}$ \\
\hline $\begin{array}{l}\text { Andrade et } \\
\text { al., } 2008^{10}\end{array}$ & $\begin{array}{l}\text { Cad Saúde } \\
\text { Pública }\end{array}$ & Brasil, 2005 & $\begin{array}{l}\text { Todos os } \\
\text { municípios } \\
\text { brasileiros }\end{array}$ & - & $\begin{array}{l}8,1 \% \text { (Brasil) } \\
9,0 \% \text { (Sudeste) } \\
8,4 \% \text { (Sul) } \\
7,5 \% \text { (Centro-oeste) } \\
7,4 \% \text { (Nordeste) } \\
6,8 \% \text { (Norte) }\end{array}$ & - \\
\hline $\begin{array}{l}\text { Carniel et al., } \\
2008^{3}\end{array}$ & $\begin{array}{l}\text { Rev Bras } \\
\text { Epidemiol }\end{array}$ & $\begin{array}{l}\text { Campinas } \\
(\mathrm{SP}), 2001\end{array}$ & $\begin{array}{l}\text { Todas as crianças } \\
\text { nascidas e residen- } \\
\text { tes no município }\end{array}$ & - & $9,1 \%$ & $\begin{array}{l}\text { i) Análise univariada: } \\
\text { - Idade materna } \\
\text { - Escolaridade da mãe } \\
\text { - Ocupação } \\
\text { - Sexo da criança } \\
\text { - Tipo de gravidez } \\
\text { - Filhos nascidos vivos } \\
\text { - Número de consultas de } \\
\text { pré-natal } \\
\text { ii) Regressão logística } \\
\text { múltipla: } \\
\text { - Tipo de gravidez } \\
\text { - Sexo da criança } \\
\text { - Número de consultas de } \\
\text { pré-natal }\end{array}$ \\
\hline $\begin{array}{l}\text { Uchimura et } \\
\text { al., } 2008^{15}\end{array}$ & $\begin{array}{l}\text { Rev Gaúcha } \\
\text { Enferm }\end{array}$ & $\begin{array}{l}\text { Maringá (PR), } \\
2001\end{array}$ & $\begin{array}{l}\text { Todas as } \\
\text { crianças nascidas no } \\
\text { município }\end{array}$ & Gravidez única & $7,6 \%$ & $\begin{array}{l}\text { - Sexo da criança } \\
\text { - Idade materna } \\
\text { - Paridade } \\
\text { - Número de consultas de } \\
\text { pré-natal } \\
\text { - Tipo de parto } \\
\text { - Duração da gestação }\end{array}$ \\
\hline
\end{tabular}


Tabela 2: Continuação

\begin{tabular}{|c|c|c|c|c|c|c|}
\hline \multirow[b]{2}{*}{ Autor, ano } & \multirow{2}{*}{$\begin{array}{l}\text { Revista de } \\
\text { publicaçáo }\end{array}$} & \multirow{2}{*}{$\begin{array}{l}\text { Local, ano do } \\
\text { estudo }\end{array}$} & \multicolumn{2}{|c|}{ Metodologia } & \multicolumn{2}{|c|}{ Principais resultados } \\
\hline & & & $\begin{array}{l}\text { Populaçáo de } \\
\text { estudo }\end{array}$ & $\begin{array}{l}\text { Critérios de } \\
\text { inclusáo }\end{array}$ & Prevalência de BPN & $\begin{array}{l}\text { Variáveis associadas ao } \\
\text { BPN }\end{array}$ \\
\hline $\begin{array}{l}\text { Uchimura et } \\
\text { al., } 2007^{9}\end{array}$ & $\begin{array}{l}\text { Cienc Cuid } \\
\text { Saúde }\end{array}$ & $\begin{array}{l}\text { Maringá (PR), } \\
1996-2002\end{array}$ & $\begin{array}{l}\text { Todas as crian- } \\
\text { ças nascidas no } \\
\text { município }\end{array}$ & Gravidez única & $\begin{array}{l}\text { Variou entre } 5,6 \% \\
(1996) \text { e } 6,1 \% \\
\text { (2002), atingindo o } \\
\text { pior resultado, } 6,6 \% \text {, } \\
\text { em } 2001\end{array}$ & $\begin{array}{l}\text { - Apgar no } 1^{\circ} \text { minuto } \\
\text { - Apgar no } 5^{\circ} \text { minuto } \\
\text { - Sexo da criança } \\
\text { - Escolaridade da mãe } \\
\text { - Estado civil } \\
\text { - Idade materna }\end{array}$ \\
\hline $\begin{array}{l}\text { Friche et al., } \\
2006^{23}\end{array}$ & $\begin{array}{l}\text { Cad Saúde } \\
\text { Pública }\end{array}$ & $\begin{array}{l}\text { Belo } \\
\text { Horizonte } \\
(\mathrm{MG}), 2001\end{array}$ & $\begin{array}{l}\text { Todas as crianças } \\
\text { nascidas e residen- } \\
\text { tes no município }\end{array}$ & - & $10,6 \%$ & 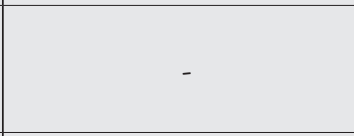 \\
\hline $\begin{array}{l}\text { Passebon et } \\
\text { al., } 2006^{24}\end{array}$ & $\begin{array}{l}\text { Cad Saúde } \\
\text { Colet } \\
\text { (Rio J) }\end{array}$ & $\begin{array}{l}\text { Campos dos } \\
\text { Goytacazes } \\
(\mathrm{RJ}), 1999\end{array}$ & $\begin{array}{l}\text { Todas as crianças } \\
\text { nascidas e residen- } \\
\text { tes no município }\end{array}$ & - & $7,5 \%$ & - \\
\hline $\begin{array}{l}\text { Mascarenhas } \\
\text { et al., } 2006^{25}\end{array}$ & $\begin{array}{l}\text { Rev Bras } \\
\text { Saúde } \\
\text { Matern } \\
\text { Infant }\end{array}$ & $\begin{array}{l}\text { Piripiri (PI), } \\
2000-2002\end{array}$ & $\begin{array}{l}\text { Todas as crianças } \\
\text { nascidas e residen- } \\
\text { tes no município }\end{array}$ & - & $\begin{array}{l}\text { Variou entre } 3,8 \% \\
(2000) \text { e } 4,6 \% \\
(2002)\end{array}$ & - \\
\hline $\begin{array}{l}\text { Giglio et al., } \\
2005^{16}\end{array}$ & $\begin{array}{l}\text { Rev Bras } \\
\text { Ginecol }\end{array}$ & $\begin{array}{l}\text { Goiânia } \\
(\mathrm{GO}), 2000\end{array}$ & $\begin{array}{l}\text { Todas as crianças } \\
\text { nascidas e residen- } \\
\text { tes no município }\end{array}$ & $\begin{array}{l}\text { Idade gesta- } \\
\text { cional } \geq 22 \\
\text { semanas } \\
\text { Peso ao nascer } \\
\geq 500 \mathrm{~g}\end{array}$ & $6,9 \%$ & $\begin{array}{l}\text { i) Análise univariada: } \\
\text { - Idade materna } \\
\text { - Número de consultas de } \\
\text { pré-natal } \\
\text { - Tipo de hospital do } \\
\text { parto } \\
\text { - Duração da gestação } \\
\text { - Sexo da criança } \\
\text { - Malformação congênita } \\
\text { ii) Análise de regressáo } \\
\text { logística: } \\
\text { Modelo a) } \\
\text { - Duraçấo da gestação } \\
\text { - Malformação congênita } \\
\text { - Categoria de hospital de } \\
\text { nascimento } \\
\text { - Tipo de hospital do } \\
\text { parto } \\
\text { - Sexo da criança } \\
\text { Modelo b) } \\
\text { - Categoria de hospital de } \\
\text { nascimento } \\
\text { - Região de residência da } \\
\text { mãe } \\
\text { - Sexo da criança } \\
\text { - Número de consultas de } \\
\text { pré-natal } \\
\text { - Idade materna }\end{array}$ \\
\hline
\end{tabular}


Tabela 2: Continuação

\begin{tabular}{|c|c|c|c|c|c|c|}
\hline \multirow[b]{2}{*}{ Autor, ano } & \multirow{2}{*}{$\begin{array}{l}\text { Revista de } \\
\text { publicaçáo }\end{array}$} & \multirow{2}{*}{$\begin{array}{l}\text { Local, ano do } \\
\text { estudo }\end{array}$} & \multicolumn{2}{|c|}{ Metodologia } & \multicolumn{2}{|c|}{ Principais resultados } \\
\hline & & & $\begin{array}{l}\text { Populaçáo de } \\
\text { estudo }\end{array}$ & $\begin{array}{l}\text { Critérios de } \\
\text { inclusão }\end{array}$ & Prevalência de BPN & $\begin{array}{l}\text { Variáveis associadas ao } \\
\text { BPN }\end{array}$ \\
\hline $\begin{array}{l}\text { Minamisawa } \\
\text { et al., } 2004^{5}\end{array}$ & $\begin{array}{l}\text { Revista Elet } \\
\text { Enf }\end{array}$ & Goiás, 2000 & $\begin{array}{l}\text { Todas as crianças } \\
\text { nascidas e residen- } \\
\text { tes no estado }\end{array}$ & Gravidez única & $5,96 \%$ & $\begin{array}{l}\text { i) Análise bivariada: } \\
\text { - Idade materna } \\
\text { - Escolaridade da mãe } \\
\text { - Estado civil } \\
\text { - Paridade } \\
\text { - Número de consultas de } \\
\text { pré-natal } \\
\text { - Duração da gestação } \\
\text { - Tipo de parto } \\
\text { - Local de ocorrência do } \\
\text { parto } \\
\text { - Sexo da criança } \\
\text { - Raça/cor da criança } \\
\text { ii) Regressão logística: } \\
\text { - Idade materna } \\
\text { - Estado civil } \\
\text { - Escolaridade da mãe } \\
\text { - Número de consultas de } \\
\text { pré-natal } \\
\text { - Local de ocorrência do } \\
\text { parto } \\
\text { - Sexo da criança } \\
\text { - Duração da gestação } \\
\text { - Tipo de parto }\end{array}$ \\
\hline $\begin{array}{l}\text { Silva, Barros, } \\
2004^{17}\end{array}$ & $\begin{array}{l}\text { Acta Paul } \\
\text { Enf }\end{array}$ & \begin{tabular}{|l} 
Presidente \\
Prudente (SP), \\
$1998-2001$ \\
\end{tabular} & $\begin{array}{l}\text { Todas as crianças } \\
\text { nascidas e residen- } \\
\text { tes no município }\end{array}$ & - & $7,2 \%$ & 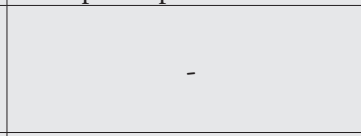 \\
\hline $\begin{array}{l}\text { Guimarães, } \\
\text { Velásquez- } \\
\text { Meléndez, } \\
2002^{7}\end{array}$ & $\begin{array}{l}\text { Rev Bras } \\
\text { Saúde } \\
\text { Matern } \\
\text { Infant }\end{array}$ & $\begin{array}{l}\text { Itaúna (MG), } \\
1997 \text { - } 1999\end{array}$ & $\begin{array}{l}\text { Todas as crianças } \\
\text { nascidas e residen- } \\
\text { tes no município }\end{array}$ & $\begin{array}{l}\text { Gravidez única } \\
\text { Preenchimento } \\
\text { da informação } \\
\text { sobre o tipo } \\
\text { de gravidez } \\
\text { Nascimentos } \\
\text { hospitalares }\end{array}$ & $7,0 \%$ & $\begin{array}{l}\text { i) Análise bivariada: } \\
\text { - Duração da gestação } \\
\text { - Número de consultas de } \\
\text { pré-natal } \\
\text { - Escolaridade da mãe } \\
\text { - Idade materna } \\
\text { ii) Regressão logística: } \\
\text { - Sexo da criança } \\
\text { - Duração da gestação } \\
\text { - Escolaridade da mãe } \\
\text { - Idade materna }\end{array}$ \\
\hline $\begin{array}{l}\text { Costa et al., } \\
2002^{18}\end{array}$ & $\begin{array}{l}\text { Cad Saúde } \\
\text { Publica }\end{array}$ & $\begin{array}{l}\text { Feira de } \\
\text { Santana (BA), } \\
1998\end{array}$ & $\begin{array}{l}\text { Todas as crian- } \\
\text { ças nascidas no } \\
\text { município }\end{array}$ & $\begin{array}{l}\text { Mães } \\
\text { adolescentes } \\
\text { (10-19 anos) } \\
\text { Mães adultas } \\
\text { jovens } \\
\text { (20-24 anos) }\end{array}$ & $9,7 \%$ & - Idade materna \\
\hline
\end{tabular}


Tabela 2: Continuação

\begin{tabular}{|c|c|c|c|c|c|c|}
\hline \multirow[b]{2}{*}{ Autor, ano } & \multirow{2}{*}{$\begin{array}{l}\text { Revista de } \\
\text { publicaçáo }\end{array}$} & \multirow{2}{*}{$\begin{array}{l}\text { Local, ano do } \\
\text { estudo }\end{array}$} & \multicolumn{2}{|c|}{ Metodologia } & \multicolumn{2}{|c|}{ Principais resultados } \\
\hline & & & $\begin{array}{l}\text { Populaçáo de } \\
\text { estudo }\end{array}$ & $\begin{array}{l}\text { Critérios de } \\
\text { inclusáo }\end{array}$ & Prevalência de BPN & $\begin{array}{l}\text { Variáveis associadas ao } \\
\text { BPN }\end{array}$ \\
\hline $\begin{array}{l}\text { Nascimento, } \\
\text { Gotlieb, } \\
2001^{19}\end{array}$ & $\begin{array}{l}\text { Inf } \\
\text { Epidemiol } \\
\text { SUS }\end{array}$ & $\begin{array}{l}\text { Guaratinguetá } \\
\text { (SP), } 1998\end{array}$ & $\begin{array}{l}\text { Todas as crian- } \\
\text { ças nascidas no } \\
\text { município }\end{array}$ & $\begin{array}{l}\text { Incluídas crian- } \\
\text { ças nascidas de } \\
\text { gravidez única } \\
\text { e que apresen- } \\
\text { taram dados } \\
\text { sobre o peso ao } \\
\text { nascer } \\
\end{array}$ & $8,2 \%$ & $\begin{array}{l}\text { - Tipo de parto } \\
\text { - Paridade } \\
\text { - Escolaridade da mãe } \\
\text { - Número de consultas de } \\
\text { pré-natal } \\
\text { - Idade materna } \\
\text { - Duração da gestação }\end{array}$ \\
\hline $\begin{array}{l}\text { Costa, } \\
\text { Gotlieb, } \\
1998^{20}\end{array}$ & $\begin{array}{l}\text { Rev Saúde } \\
\text { Pública }\end{array}$ & \begin{tabular}{|l|} 
Itararé \\
Marília \\
Pariquera-Açu \\
Santo André \\
São José dos \\
Campos \\
(SP), janeiro a \\
julho de 2002 \\
(exceto abril) \\
\end{tabular} & $\begin{array}{l}\text { Todas as crian- } \\
\text { ças nascidas nos } \\
\text { municípios }\end{array}$ & $\begin{array}{l}\text { Gravidez única } \\
\text { Nascimentos } \\
\text { hospitalares }\end{array}$ & $\begin{array}{l}\text { 10,4\% (Itararé) } \\
\text { 7,8\% (São José dos } \\
\text { Campos) } \\
\text { 7,4\% (Marília) } \\
\text { 6,8\% (Santo André) } \\
\text { 6,6\%(Pariquera-Açu) }\end{array}$ & $\begin{array}{l}\text { - Sexo da criança } \\
\text { - Duração da gestação } \\
\text { - Idade materna } \\
\text { - Paridade }\end{array}$ \\
\hline $\begin{array}{l}\text { Tiaraju, } \\
\text { Wisbec, } \\
1998^{21}\end{array}$ & $\begin{array}{l}\text { Inf } \\
\text { Epidemiol } \\
\text { SUS } \\
\end{array}$ & $\begin{array}{l}\begin{array}{l}\text { Blumenau } \\
(\mathrm{SC})\end{array} \\
1994-1997 \\
\end{array}$ & $\begin{array}{l}\text { Todas as crianças } \\
\text { nascidas e residen- } \\
\text { tes no município }\end{array}$ & - & $\begin{array}{l}\text { Variou entre } 8,7 \% \\
(1994) \text { e } 6,8 \% \\
(1997)\end{array}$ & - \\
\hline Maia, $1997^{22}$ & $\begin{array}{l}\text { Rev Saúde } \\
\text { Pública }\end{array}$ & $\begin{array}{l}\text { Passos (MG), } \\
\text { abril de } 1995 \\
\text { a abril de } \\
1996 \\
\end{array}$ & $\begin{array}{l}\text { Todas as crian- } \\
\text { ças nascidas no } \\
\text { município }\end{array}$ & - & $10,0 \%$ & - \\
\hline $\begin{array}{l}\text { Bicalho- } \\
\text { Mariotoni, } \\
\text { Barros Filho, } \\
1997^{26}\end{array}$ & $\begin{array}{l}\text { Rev Paul } \\
\text { Pediatria }\end{array}$ & $\begin{array}{l}\text { Campinas } \\
(\mathrm{SP}), 1995\end{array}$ & $\begin{array}{l}\text { Todas as crian- } \\
\text { ças nascidas no } \\
\text { município }\end{array}$ & - & $\begin{array}{l}10,0 \% \text { (população } \\
\text { total) } \\
8,3 \% \text { (gravidez } \\
\text { única) }\end{array}$ & - \\
\hline $\begin{array}{l}\text { Rodrigues et } \\
\text { al., } 1994^{27}\end{array}$ & $\begin{array}{l}\text { Rev Méd } \\
\text { Minas } \\
\text { Gerais } \\
\end{array}$ & $\begin{array}{l}\text { Belo } \\
\text { Horizonte } \\
(\mathrm{MG}), 1992 \\
\end{array}$ & \begin{tabular}{|l|} 
Todas as crianças \\
nascidas e residen- \\
tes no município \\
\end{tabular} & - & $10,1 \%$ & - \\
\hline \multicolumn{7}{|c|}{ Estudos de tendência secular } \\
\hline $\begin{array}{l}\text { Próspero et } \\
\text { al., } 2008^{28}\end{array}$ & $\begin{array}{l}\text { Saúde } \\
\text { Coletiva }\end{array}$ & $\begin{array}{l}\text { Itajaí (SC), } \\
1996-2002\end{array}$ & $\begin{array}{l}\text { Todas as crianças } \\
\text { nascidas no municí- } \\
\text { pio e residentes na } \\
\text { zona urbana }\end{array}$ & Gravidez única & $\begin{array}{l}\text { Variou entre } 8,8 \% \\
(1996) \text { e } 10,0 \% \\
\text { (2002), atingindo } \\
\text { o melhor resultado, } \\
7,32 \% \text {, em } 2001\end{array}$ & $\begin{array}{l}\text { Análise dos fatores associa- } \\
\text { dos à evoluçáo do BPN } \\
\text { - Duração da gestaçáo }\end{array}$ \\
\hline $\begin{array}{l}\text { Vidal et al., } \\
2005^{29}\end{array}$ & $\begin{array}{l}\text { Rev Assoc } \\
\text { Med Bras }\end{array}$ & $\begin{array}{l}\text { Recife (PE), } \\
\text { julho de } 1991 \\
\text { a dezembro de } \\
2000\end{array}$ & $\begin{array}{l}\text { Todas as crianças } \\
\text { nascidas na mater- } \\
\text { nidade do IMIP }\end{array}$ & - & $\begin{array}{l}\text { Variou entre } 17,8 \% \\
\text { (1991) e } 18,7 \% \\
\text { (2000), atingindo } \\
\text { um patamar de } \\
22,8 \% \text { em } 2000 \text { e } \\
\text { um aumento gra- } \\
\text { dativo ao longo dos } \\
\text { anos, principalmente } \\
\text { a partir de } 1997\end{array}$ & - \\
\hline
\end{tabular}


Tabela 2: Continuação

\begin{tabular}{|c|c|c|c|c|c|c|}
\hline \multirow[b]{2}{*}{ Autor, ano } & \multirow{2}{*}{$\begin{array}{l}\text { Revista de } \\
\text { publicaçáo }\end{array}$} & \multirow{2}{*}{$\begin{array}{l}\text { Local, ano do } \\
\text { estudo }\end{array}$} & \multicolumn{2}{|c|}{ Metodologia } & \multicolumn{2}{|c|}{ Principais resultados } \\
\hline & & & $\begin{array}{l}\text { Populaçáo de } \\
\text { estudo }\end{array}$ & $\begin{array}{l}\text { Critérios de } \\
\text { inclusáo }\end{array}$ & Prevalência de BPN & $\begin{array}{l}\text { Variáveis associadas ao } \\
\text { BPN }\end{array}$ \\
\hline $\begin{array}{l}\text { Monteiro et } \\
\text { al., } 2000^{6}\end{array}$ & $\begin{array}{l}\text { Rev Saúde } \\
\text { Pública }\end{array}$ & $\begin{array}{l}\text { São Paulo, } \\
1976-1998 \\
\text { (os dados } \\
\text { do Sinasc } \\
\text { correspondem } \\
\text { ao período de } \\
1993-1998 \text { ) }\end{array}$ & $\begin{array}{l}\text { Todas as crian- } \\
\text { ças nascidas no } \\
\text { município }\end{array}$ & - & $\begin{array}{l}\text { Variou entre } 9,4 \% \\
\text { (1993) e 8,9\% (1998), } \\
\text { mostrando variaçóes } \\
\text { anuais pequenas }\end{array}$ & $\begin{array}{l}\text { i) Análise dos fatores } \\
\text { associados ao BPN } \\
\text { - Escolaridade da mãe } \\
\text { - Idade materna } \\
\text { - Paridade } \\
\text { - Duraçáo da gestação } \\
\text { ii) Análise dos fatores } \\
\text { associados à evolução do } \\
\text { BPN } \\
\text { - Duração da gestação } \\
\text { - Paridade } \\
\text { - Idade materna }\end{array}$ \\
\hline
\end{tabular}

BPN: Baixo Peso ao Nascer.

de pré-natal ${ }^{1,3,5,7,14-16,19}$, sexo da criança $a^{1,3,5,7,9,15,16,20}$, duração da gestação ${ }^{1,5-7,15,16,19,20,28}$ e escolaridade da mãe $\mathrm{e}^{1,3,5-7,9,19}$

No estudo realizado por Andrade et al. ${ }^{10}$ no Brasil, no ano 2005, os autores agruparam o país por regiáo geográfica e por tamanho da população dos municípios (50 mil habitantes como referência), analisando as desigualdades segundo esse agrupamento. Observaram-se desigualdades do BPN para as variáveis escolaridade da mãe, prematuridade e número de consultas de pré-natal, assim como maiores prevalências de BPN, partos cesáreos e prematuridade nos municípios com 50 mil habitantes ou mais.

Por outro lado, o estudo de tendência realizado no município de São Paulo ${ }^{6}$ indica evidências de evolução desigual e inversa do peso ao nascer segundo o nível socioeconômico da população. Os autores atribuem a evolução favorável do BPN nas camadas de mais baixo nível socioeconômico ao melhor desempenho do crescimento intrauterino, que poderia decorrer de melhorias das condiçóes econômicas, no peso e na altura das gestantes, na assistência pré-natal e, possivelmente, do declínio no hábito de fumar. $\mathrm{O}$ artigo, ainda, advoga que a evolução desfavorável do peso ao nascer nos estratos de alto nível socioeconômico é devida, aparentemente, ao aumento na frequência de recém-nascidos prematuros, tendência provocada por fatores ainda não conhecidos.

Os estudos foram classificados, na maioria, como de qualidade alta $(n=17)^{1,3,5-7,9,10,15-20,24,26,28,29}$. Os outros seis estudos ${ }^{14,21-23,25,27}$ apresentaram qualidade média. As principais limitaçôes dos estudos, segundo os critérios de Downs \& Black $^{11}$, foram a não descrição das razóes de perda de participantes e a não apresentação e controle dos principais fatores de confusão. $\mathrm{O}$ controle adequado das variáveis de confusão na análise de associações foi atingido por sete estudos ${ }^{1,3,5-7,16,28}$, os quais foram considerados de qualidade alta. Com relação à validade externa, todos os artigos foram representativos e passíveis de generalização, vantagem das pesquisas com dados dos Sistemas de Informação em Saúde (SIS).

\section{Discussão}

A disponibilização, no site da Internet do Departamento de Informática do SUS (Datasus), dos dados dos SIS, desenvolvidos e implantados pelo Ministério da Saúde em todo o Brasil e desagregados até o nível dos municípios, constitui uma ferramenta muito importante para análises epidemiológicas ${ }^{30}$. As informaçóes contidas nos SIS adquiriram valor ao serem cada vez mais necessárias para as formulações e avaliaçóes de políticas públicas voltadas para a melhoria das condiçóes de saúde da população. Uma das maiores vantagens dessa utilização é permitir aos pesquisadores a realização, com baixo custo, de estudos de base populacional e com abrangência nacional, o que possibilita também novas formas de análise da situação de saúde e de avaliação dos serviços ${ }^{30,31}$. Nesse contexto, o Sinasc tem sido utilizado para traçar o perfil dos nascidos vivos e o comportamento do BPN e da prematuridade ${ }^{30}$.

Deste modo, os resultados apresentados no presente artigo são de grande importância para o aprofundamento do BPN como indicador de saúde materno-infantil e permitem estabelecer o perfil epidemiológico populacional, as comparaçóes socioespaciais e explicaçóes 
Tabela 3: Estratificação dos municípios brasileiros com estudos sobre baixo peso ao nascer, baseado nos dados do Sistema de Informações sobre Nascidos Vivos (Sinasc), segundo localização geográfica, porte populacional e índice de desenvolvimento humano do município (IDH-M)

\begin{tabular}{|c|c|c|c|}
\hline \multirow{2}{*}{$\begin{array}{l}\text { Porte Populacional } \\
\text { (habitantes) }\end{array}$} & \multicolumn{3}{|c|}{ Localizaçáo geográfica: Nordeste } \\
\hline & $\begin{array}{c}\text { IDH-M elevado } \\
(0,800-0,899)\end{array}$ & $\begin{array}{l}\text { IDH-M médio } \\
(0,500-0,799)\end{array}$ & $\begin{array}{l}\text { IDH-M baixo } \\
\quad(<0,500)\end{array}$ \\
\hline Pequeno $(<20.000)$ & $\mathrm{BPN}$ ? & $\mathrm{BPN}$ ? & $\mathrm{BPN}$ ? \\
\hline Médio (20.000-100.000) & $\mathrm{BPN}$ ? & Piripiri* & $\mathrm{BPN}$ ? \\
\hline Grande (100.000-500.000) & $\mathrm{BPN}$ ? & Feira de Santana & $\mathrm{BPN}$ ? \\
\hline Muito grande (> 500.000) & $\mathrm{BPN}$ ? & $\mathrm{BPN}$ ? & $\mathrm{BPN}$ ? \\
\hline \multirow{2}{*}{$\begin{array}{l}\text { Porte Populacional } \\
\text { (habitantes) }\end{array}$} & \multicolumn{3}{|c|}{ Localizaçáo geográfica: Centro-oeste } \\
\hline & $\begin{array}{c}\text { IDH-M elevado } \\
(0,800-0,899)\end{array}$ & $\begin{array}{c}\text { IDH-M médio } \\
(0,500-0,799)\end{array}$ & $\begin{array}{c}\text { IDH-M baixo } \\
\quad(<0,500)\end{array}$ \\
\hline Pequeno $(<20.000)$ & $\mathrm{BPN}$ ? & $\mathrm{BPN}$ ? & BPN? \\
\hline Médio (20.000-100.000) & $\mathrm{BPN}$ ? & $\mathrm{BPN}$ ? & $\mathrm{BPN}$ ? \\
\hline Grande (100.000-500.000) & $\mathrm{BPN}$ ? & $\mathrm{BPN}$ ? & $\mathrm{BPN}$ ? \\
\hline Muito grande (> 500.000) & Goiânia & $\mathrm{BPN}$ ? & $\mathrm{BPN}$ ? \\
\hline \multirow{2}{*}{$\begin{array}{l}\text { Porte Populacional } \\
\text { (habitantes) }\end{array}$} & \multicolumn{3}{|c|}{ Localizaçáo geográfica: Sudeste } \\
\hline & $\begin{array}{c}\text { IDH-M elevado } \\
(0,800-0,899)\end{array}$ & $\begin{array}{c}\text { IDH-M médio } \\
(0,500-0,799)\end{array}$ & $\begin{array}{c}\text { IDH-M baixo } \\
(<0,500)\end{array}$ \\
\hline Pequeno $(<20.000)$ & $\mathrm{BPN}$ ? & Pariquera-Açu & $\mathrm{BPN}$ ? \\
\hline Médio (20.000-100.000) & Itaúna* & $\begin{array}{c}\text { Passos* } \\
\text { Itararé** }\end{array}$ & $\mathrm{BPN}$ ? \\
\hline Grande (100.000-500.000) & $\begin{array}{c}\text { Presidente Prudente } \\
\text { Marília } \\
\text { Guaratinguetá }\end{array}$ & Campos dos Goytacazes & $\mathrm{BPN}$ ? \\
\hline Muito grande $(>500.000)$ & $\begin{array}{c}\text { Campinas } \\
\text { São José dos Campos } \\
\text { Rio de Janeiro } \\
\text { Santo André } \\
\text { Sáo Paulo } \\
\text { Belo Horizonte }\end{array}$ & $\mathrm{BPN}$ ? & $\mathrm{BPN}$ ? \\
\hline & & alização geográfica: Sul & \\
\hline (habitantes) & $\begin{array}{c}\text { IDH-M elevado } \\
(0,800-0,899)\end{array}$ & $\begin{array}{l}\text { IDH-M médio } \\
(0,500-0,799)\end{array}$ & $\begin{array}{l}\text { IDH-M baixo } \\
\quad(<0,500)\end{array}$ \\
\hline Pequeno $(<20.000)$ & $\mathrm{BPN}$ ? & $\mathrm{BPN} ?$ & $\mathrm{BPN}$ ? \\
\hline Médio (20.000-100.000) & $\mathrm{BPN}$ ? & $\mathrm{BPN}$ ? & $\mathrm{BPN}$ ? \\
\hline Grande (100.000-500.000) & $\begin{array}{l}\text { Blumenau } \\
\text { Itajaí } \\
\text { Maringá }\end{array}$ & $\mathrm{BPN}$ ? & $\mathrm{BPN}$ ? \\
\hline Muito grande (> 500.000) & $\mathrm{BPN}$ ? & $\mathrm{BPN}$ ? & $\mathrm{BPN}$ ? \\
\hline
\end{tabular}

Fontes:

- Programa das Naçóes Unidas para o Desenvolvimento. Atlas do Desenvolvimento Humano, 2000.

- Instituto Brasileiro de Geografia e Estatística. Censo Demográfico. 2000.

${ }^{*}=$ Categoria IDH-M muito elevado $(>0,900)$ não mostrada por existirem somente dois municípios no Brasil nesta categoria.

* = Município de mais de 50.000 habitantes.

** $=$ Município de menos de 50.000 habitantes.

BPN $?=$ Nenhum município com estudo sobre baixo peso ao nascer baseado nos dados do Sinasc. 
causais. Essas informações são as bases para uma ação preventiva mais apropriada.

Não obstante, é necessário ressaltar que, considerando o acesso gratuito e em meio eletrônico aos dados do Sinasc, esperava-se uma maior quantidade de trabalhos publicados, principalmente de tendência secular. A análise secular do peso ao nascer possibilita a definição objetiva de uma situação desejada a ser atingida. $\mathrm{O}$ estudo de evolução da distribuição do peso ao nascer pode contribuir para compreender a realidade - percebida dinamicamente -, quando esta é analisada em termos de mudanças e seus fatores de risco ao longo do tempo, o que tornam possíveis formulações de hipóteses etiológicas mais consistentes ${ }^{32}$.

As revistas nas quais os artigos foram publicados abrangem diversas áreas de estudo como epidemiologia, serviços de saúde, enfermagem, saúde pública, ginecologia, saúde materno-infantil, o que configura o BPN como assunto de interesse multidisciplinar e multiprofissional. As publicaçôes nacionais veicularam 100\% do total de artigos selecionados. Estudo sobre a utilização de dados secundários do SIM, Sinasc e SIH na produção científica brasileira apontou que a maior parte é publicada em língua portuguesa, em periódicos nacionais e tendo a Revista de Saúde Pública e os Cadernos de Saúde Pública entre os mais utilizados ${ }^{30}$.

O âmbito municipal, como espaço geográfico privilegiado das análises, responde à necessidade de se abordar a situação de saúde em níveis cada vez mais desagregados que considerem a descentralização das açóes de saúde. A predominância da esfera municipal como local de estudo, além de representar a possibilidade de intervençóes específicas a serem adotadas pelos níveis locais, pode ser útil para a extrapolação de resultados. Nos estudos municipais em que a população total é considerada como amostra, a aplicaçáo de testes de inferência estatística permite generalizar os dados do município estudado para outras localidades de características semelhantes. Para esses fins, critérios de estratificação dos municípios brasileiros devem ser adotados, propiciando também apreciaçóes sobre o comportamento da produção científica.

Adotando as características de localização geográfica, porte populacional e índice de desenvolvimento humano do município (IDH-M) como critérios de estratificação, as diferentes situaçóes em que os municípios estudados podem ser enquadrados são mostradas na tabela 3. A análise a respeito expressa a concentração da produção científica no Sudeste, notadamente São Paulo, corroborando os resultados obtidos por pesquisas em outros temas de saúde ${ }^{30,33}$. Este fato pode estar relacionado ao maior número de instituiçóes de ensino superior nesta região do país. Ressalta-se que nenhum dos estudos analisou a regiāo Norte, havendo apenas dois municípios do Nordeste ${ }^{18,25}$ e um do Centrooeste $^{16}$. Referente ao IDH-M, chama a atenção também a existência de uma concentração de estudos nos município com IDH-M elevado, não havendo estudo algum em município com IDH-M baixo. Esses dados são importantes, pois indicam a necessidade de estudos em municípios com IDH-M médio e baixo, sobretudo naqueles de pequeno, médio e grande porte populacional, uma vez que, no Brasil, em 2002, apenas 33 municípios eram de porte populacional muito grande, representando uma ínfima parcela do país ${ }^{34}$. O comportamento do BPN nestes municípios pouco explorados deve ter especificidades relevantes que necessitam ser conhecidas.

$\mathrm{O}$ BPN assume taxas suficientemente altas a ponto de requerer medidas de saúde pública imediata quando ocorrem em $15 \%$ dos nascidos vivos ${ }^{8}$. Este ponto crítico não foi alcançado por qualquer dos municípios estudados, variando entre $5,6 \%{ }^{5}$ e $10,6 \%{ }^{23}$, nem nas diferentes regiōes do Brasil ${ }^{10}$. A maior prevalência de BPN no Brasil foi de $10,4 \%$ no ano $1996^{35}$. Este resultado foi estimado em um estudo que agrupou 62 inquéritos nacionais de demografia e saúde em países em desenvolvimento entre 1990 e 2000, após ajustes que consideram a subestimação das prevalências de BPN em todos os países estudados.

Se analisarmos o problema do BPN no Brasil, utilizando as taxas de prevalência anteriores, sua prevenção parece não representar uma prioridade. Porém, estratégias para a prevenção não deveriam ser subestimadas, se considerarmos as condiçóes em que nascem as crianças prematuras ou com RCIU e suas consequências para a saúde, assim como os possíveis desfechos negativos avaliados sob a perspectiva conceitual do ciclo da vida. A prevenção do BPN apresenta um enorme impacto na saúde, na produtividade dos indivíduos e na sociedade como um todo, pois contribui para a ruptura do ciclo intergeraçóes de pobreza, desnutrição e doenças que se perpetua pela presença de recém-nascidos com peso inferior a 2.500 gramas. Seguindo este raciocínio, recém-nascidos do sexo feminino, nascidos com BPN, apresentam uma maior predisposição de serem meninas de baixa estatura e podem se tornar mulheres desnutridas com um maior risco de ter filhos com BPN - mantendo-se assim um círculo vicioso cujo efeito final seria a manutenção das altas taxas de BPN de geração em geração $0^{8,36,37}$.

Descobertas feitas no Brasil e em outros países como Estados Unidos e Noruega ${ }^{10,38-40}$ apontam o paradoxo do BPN, com percentuais mais baixos nas localidades de menos desenvolvimento socioeconômico. Várias hipóteses explicativas para o paradoxo do BPN têm sido geradas por diferentes pesquisadores. As explicaçóes para o paradoxo do BPN compreendem a subnotificação de nascidos vivos, principalmente em municípios de menor porte populacional e mais atrasados; 
os nascimentos por partos cesáreos e os estilos de vida, incluindo o estresse, os comportamentos nutricionais inadequados e o tabagismo durante a gestação $\mathrm{o}^{10,40,41}$. A principal explicação para este paradoxo baseia-se na tendência de aumento da prematuridade, fato observado no Brasil e em suas regióes, com particularidades nas regiôes Norte e Nordeste que apresentam diminuição; porém, sendo observado aumento na maior parte das capitais ${ }^{10,40,42,43}$. Indica-se que as diferenças regionais nas taxas de BPN no país parecem estar mais relacionadas à disponibilidade de assistência perinatal do que às condições sociais ${ }^{44}$. Nenhum comportamento sobre as desigualdades do BPN pode ser traçado a partir dos estudos incluídos nesta revisão. A pequena quantidade de municípios estudados e a maior concentração no Sudeste - do IDH-M elevado e para os portes populacionais grande e muito grande - explica esta restrição.

É reconhecido que o BPN apresenta etiologia multicausal. Em metanálise ${ }^{45}$ sobre a determinação do peso ao nascer, duração da gestação e RCIU, 43 determinantes potenciais foram identificados e avaliados. Nos países em desenvolvimento, os principais fatores estabelecidos com efeito sobre o crescimento intrauterino foram: raça/etnia, estatura da mãe, peso pré-gestacional, ganho de peso gestacional/consumo energético, infecção por malária. Outros fatores como sexo da criança, peso do pai, estatura do pai, peso da mãe ao nascer, paridade, história anterior de baixo peso ao nascer, tabagismo e consumo de álcool também foram identificados como importantes. No que se refere à duração gestacional, os fatores de maior sistematização com efeitos causais bem definidos foram o peso pré-gestacional, a história prévia de prematuridade ou aborto espontâneo, a exposição in utero ao dietilestilbestrol e o tabagismo. Estudos sobre a associação da adequação do pré-natal ${ }^{46} \mathrm{e}$ da ansiedade ${ }^{2}$ com o peso ao nascer também foram sistematizados, concluindo que as evidências sobre tais associaçóes são controversas, o que demonstra a necessidade de maior quantidade de estudos cuidadosamente desenhados.

Observa-se que variáveis muito importantes na determinaçáo do BPN náo formam parte do banco de dados do Sinasc. Destacam-se, principalmente, condiçóes modificáveis representadas por variáveis nutricionais e de estilos de vida (tabagismo, etilismo). Sendo assim, ressalta-se a importância da complementaridade dos resultados sobre a etiologia do BPN obtidos com a utilização do Sinasc.

Os resultados sistematizados nesta revisão são consistentes para a avaliação das associaçóes entre a adequação do pré-natal, a idade materna e a instrução materna com o peso ao nascer. Nestas análises prevaleceram os artigos com adequado controle das variáveis de confusão. A plausibilidade desses achados é ilustrada através das relaçóes lógicas estabelecidas entre as variáveis na determinação do BPN, que destacam a adequação da assistência pré-natal (determinante intermediário) e a instrução materna (determinante distal) como importantes condiçóes dos determinantes proximais do peso ao nascer (duração da gestação e velocidade de crescimento intrauterino) $)^{6}$. O sexo da criança também mostra resultados consistentes comparados aos obtidos em revisão anterior ${ }^{45}$.

O estabelecimento da importância relativa dos múltiplos fatores de risco que determinam a prevalência de $\mathrm{BPN}$, assim como a magnitude desses efeitos, constitui, ainda, prioridade científica. A prevenção do $\mathrm{BPN}$, avaliando-se a distribuição devida ao RCIU e ao nascimento pré-termo, assim como a identificação dos fatores modificáveis por intervençôes sociais de saúde e nutrição, deve constituir o alvo das intervençóes destinadas a um melhor impacto na saúde materno-infantil. Nesse sentido, observa-se um aumento crescente na proporção de partos prematuros no Brasil ${ }^{42,43}$, associado à maior ocorrência de baixo peso ao nascer ${ }^{47}$. $\mathrm{O}$ aumento das taxas de cesáreas tem sido identificado como um importante fator relacionado à prematuridade ${ }^{48}$, e a repetição de nascimento pré-termo em sucessivas gestações à ruptura prematura de membranas, corioamnionite, pré-eclâmpsia, trabalho de parto prematuro e intervalo intergestacional inferior a 12 meses $^{49}$.

Considerando que o uso de dados secundários para fins de pesquisa apresenta problemas de acurácia condicionados, principalmente, por erro de medição e de classificação, a utilização dos dados do Sinasc exige a avaliação da qualidade destes em termos de cobertura, completitude do preenchimento e confiabilidade das informações. Isso possibilita a obtenção de uma caracterização mais fidedigna do BPN nas diferentes localidades e o planejamento de açôes de saúde ${ }^{50-52}$.

Apesar disso, o presente estudo é de grande relevância para o entendimento do BPN no Brasil, pois: i) os recursos disponíveis muitas vezes inviabilizam o desenvolvimento de pesquisas da abrangência que os dados do Sinasc permitem ${ }^{30}$, ii) o Sinasc é um sistema caracterizado pela qualidade das informaçóes em virtude da completitude de preenchimento e consistência da informação na maioria das variáveis analisadas ${ }^{51,52}$, iii) o peso ao nascer destaca entre as variáveis de melhor completitude ${ }^{22,51}$ e consistência ${ }^{51}$. A qualidade dos dados do Sinasc também foi mostrada em análises críticas da literatura $^{50,53}$, sustentando a veracidade relacionada às taxas de baixo peso ao nascer. Muitos artigos da presente revisão apresentam resultados que especificam a qualidade dos dados utilizados. Ainda que alguns estudos apresentassem limitações do ponto de vista metodológico, outros foram bem detalhados e controlaram as confusóes por análise multivariada visualizando-se alguns fatores de risco do BPN. 


\section{Conclusões}

Apesar do seu impacto na situação de saúde e epidemiológica, o conhecimento sobre a etiologia do BPN no Brasil ainda conserva incertezas. A concretizaçáo de estudos é importante, principalmente em regióes além da Sudeste e em municípios de IDH-M médio e baixo. Os resultados apresentados permitem definir prevalências de BPN aceitáveis em todos os contextos socioespaciais, assim como a influência da idade gestacional e de outros determinantes não biológicos, como a instrução materna e a adequação do pré-natal no peso ao nascer.

\section{Referências}

1. Barbas DS, Costa AJL, Luiz RR, Kale PL. Determinantes do peso insuficiente e do baixo peso ao nascer na cidade do Rio de Janeiro, Brasil, 2001. Epidemiol Serv Saúde. 2009; 18(2):161-70.

2. Araújo DMR, Pereira NL, Kac G. Ansiedade na gestação, prematuridade e baixo peso ao nascer: uma revisão sistemática da literatura. Cad Saúde Pública. 2007; 23(4):747-56.

3. Carniel EF, Zanolli ML, Antônio MARG, Morcillo AM. Determinantes do baixo peso ao nascer a partir das declaraçóes de nascidos vivos. Rev Bras Epidemiol. 2008; 11(1):169-79.

4. World Health Organization. Recommended definitions, terminology and format for statistical tables related to the perinatal period and use of a new certificate for cause of perinatal deaths. Modifications recommended by FIGO as amended October 14, 1976. Acta Obstet Gynecol Scand. 1977; 56:247-53.

5. Minamisawa R, Barbosa MA, Malagoni L, Andraus LMS. Fatores associados ao baixo peso ao nascer no Estado de Goiás. Rev eletrônica enferm. 2004; 6(3).

6. Monteiro CA, Benício MHD, Ortiz LP. Tendência secular do peso ao nascer na cidade de São Paulo (1976-1998). Rev Saúde Pública 2000; 34(6):26-40.

7. Guimarães EAA, Velásquez-Melendez G. Determinantes do baixo peso ao nascer a partir do Sistema de Informação sobre nascidos vivos em Itaúna. Rev Bras Saúde Matern Infant. 2002; 2(3):283-90.

8. Llanos A, Uauy R, Gonzalez Cossio T, Barros A, para el Proyecto Challenges for Childhood Health and Nutrition Research in Latin America: addressing the 90/10 gap. Bajo Peso al Nacer. Bangladesh: Child Health and Nutrition Research Initiative; 2003.

9. Uchimura TT, Pelissari DM, Soares DFPP, Uchimura NS, Santana RG, Moraes CMS. Fatores de risco para o baixo peso ao nascer segundo as variáveis da máe e do recém-nascido, em Maringá-PR, no período de 1996 a 2002. Cienc Cuid Saúde. 2007; 6(1):51-8.

10. Andrade CLT, Szwarcwald CL, Castilho EA. Baixo peso ao nascer no Brasil de acordo com as informaçóes sobre nascidos vivos do Ministério da Saúde, 2005. Cad Saúde Pública. 2008; 24(11):2564-72.

11. Downs HS, Black N. The feasibility of creating a checklist for the assessment of the methodological quality both of randomised and non-randomised studies of health care interventions. J Epidemiol Community Health. 1998; 52:377-84.

12. Vandenbroucke JP, Von Elm E, Altman DG, Gøtzsche PC, Mulrow CD, Pocock SJ et al. Strengthening the reporting of observational studies in epidemiology (STROBE): explanation and elaboration. Epidemiol. 2007; 18:805-35.

13. Sanderson S, Tatt ID, Higgins JPT. Tools for assessing quality and susceptibility to bias in observational studies in epidemiology: a systematic review and annotated bibliography. Int J Epidemiol. 2007; 36:666-76.

14. Silva GF, Pelloso SM. Perfil das parturientes e seus recémnascidos atendidos em um hospital-escola do Noroeste do Estado do Paraná. Rev Esc Enferm USP. 2009; 43(1):95-102.

15. Uchimura TT, Pelissari DM, Uchimura NS. Baixo peso ao nascer e fatores associados. Rev Gaúcha Enferm. 2008; 29(1):33-8.

16. Giglio MRP, Lamounier JA, Neto OLM, César CM. Baixo peso ao nascer em coorte de recém-nascidos em GoiâniaBrasil no ano de 2000. Rev Bras Ginecol Obstet. 2005; 27(3): 130-6.

17. Silva TRSR, Barros SMO. Perfil do peso ao nascer no município de Presidente Prudente, São Paulo de 1998 a 2001. Acta Paul Enf. 2004; 17(3):292-7.

18. Costa MCO, Santos CAT, Sobrinho CLN, Freitas JO, Ferreira KASL, Silva MA, Paula PLB. Estudo dos partos e nascidos vivos de mães adolescentes e adultas jovens no Município de Feira de Santana, Bahia, Brasil, 1998. Cad Saúde Pública. 2002; 18(3):715-22.

19. Nascimento LFC, Gotlieb SLD. Fatores de Risco para o Baixo Peso ao Nascer, com Base em Informaçóes da Declaração de Nascido Vivo em Guaratinguetá, SP, no Ano de 1998. Inf Epidemiol SUS. 2001; 10(3):113-20.

20. Costa EC, Gotlieb SLD. Estudo epidemiológico do peso ao nascer a partir da Declaração de Nascidos Vivos. Rev Saúde Pública. 1998; 32(4):328-34.

21. Tiaraju E, Wisbec J. Implantação do SINASC e perfil dos nascidos vivos em Blumenau, 1994-1997. Inf Epidemiol SUS. 1998, 8(3):35-42.

22. Maia MAC. Caracterização dos nascidos vivos hospitalares no primeiro ano de implantação do subsistema de Informaçôes sobre nascidos vivos e Município de Minas Gerais, 1996. Rev Saúde Pública. 1997; 31(6).

23. Friche AAL, Caiaffa WT, César CC, Goulart LMF, Almeida MCM. Indicadores de saúde materno-infantil em Belo Horizonte, Minas Gerais, Brasil, 2001: análise dos diferenciais intra-urbanos. Cad Saúde Pública. 2006; 22(9):1955-65.

24. Passebon E, Bloch KV, Kale PL, Coeli CM. Associação entre peso ao nascer e mortalidade infantil no município 
de Campos dos Goytacazes - RJ. Cad Saúde Colet. 2006; 14(2):283-96.

25. Mascarenhas MDM, Rodrigues MTP, Monte NF. Caracterização dos partos e dos nascidos vivos em Piripiri, Piauí, Brasil. Rev Bras Saúde Matern Infant. 2006; 6(2):175-81.

26. Bicalho-Mariotoni GG, Barros Filho AA. Nascer em Campinas: análise de dados do Sinasc, 1995. Rev Paul Pediatria. 1997; 15(1):24-30.

27. Rodrigues CS, Ladeira RM, Magalhães Jr HM, Laudares S. Sistema de informaçáo sobre nascidos vivos - Sinasc a experiência de Belo Horizonte. Rev Méd Minas Gerais. 1994; 4(4):2-7.

28. Próspero EN, Barros SMO, Goldman RE. Evolução do peso ao nascer no município de Itajaí, Santa Catarina, Brasil após a implantação do sistema de informação de nascidos vivos. Saúde Coletiva. 2008; 5(21):71-5.

29. Vidal SA, Arruda BKG, Vanderlei LC, Frias PG. Avaliação da série histórica dos nascidos vivos em unidade terciária de Pernambuco-1991 a 2000. Rev Assoc Med Bras. 2005; 51(1):17-22.

30. Drumond EF, Machado CJ, Vasconcelos MR, França E. Utilização de dados secundários do SIM, Sinasc e SIH na produção científica brasileira de 1990 a 2006. R Bras Est Pop. 2009, 26 (1):7-19.

31. Paim JS. Atenção à saúde no Brasil. In: Paim JS. Desafios da saúde coletiva no século XXI. Salvador: EDUFBA, 2006.

32. Júnior IF, Monteiro CA. Estudo da tendência secular de indicadores de saúde como estratégia de investigação epidemiológica: editorial. Rev Saúde Pública. 2000; 34(Suppl 6):5-7.

33. Moreira RS, Nico LS, Tomita NE, Ruiz T. A saúde bucal do idoso brasileiro: revisão sistemática sobre o quadro epidemiológico e acesso aos serviços de saúde bucal. Cad Saúde Pública. 2005; 21(6):1665-75.

34. Instituto Brasileiro de Geografia e Estatística. Pesquisa de informaçôes básicas municipais. Perfil dos municípios brasileiros. Gestão Pública 2002. Rio de Janeiro: IBGE; 2002.

35. Blanc AK, Wardlaw T. Monitoring low birth weight: an evaluation of international estimates and an updated estimation procedure. Bull World Health Organ. 2005; 83(3):178-85.

36. United Nations, Administrative Committee on Coordination Sub-Committee on Nutrition. $4^{\text {th }}$ Report on the World Nutrition Situation: Nutrition throughout the life cycle. Genebra: ACC/SCN; 2000.

37. Instituto de Nutrición de Centro - América y Panamá / Organización Panamericana de la Salud. Nutrición y Alimentación en América Latina y El Caribe: En pro de la Seguridad Alimentaria y Nutricional: INCAP/OPS; 2000. Publicación INCAP CE/118.

38. Silva AAM, Bettiol H, Barbieri MA, Pereira MM, Brito LGO, Ribeiro VS, et al. Why are the low birthweight rates in Brazil higher in richer than in poorer municipalities? Exploring the epidemiological paradox of low birthweight. Paediatr Perinat Epidemiol. 2005; 19:43-9.
39. Melve KK, Skjaerven R. Birthweight and perinatal mortality: paradoxes, social class, and sibling dependencies. Int J Epidemiol. 2003; 32:625-32.

40. Silva AAM, Bettiol H, Barbieri MA, Brito LGO, Pereira MM, Aragão VMF, Ribeiro VS. Which factors could explain the low birth weight paradox? Rev Saúde Pública. 2006; 40(4):648-55.

41. Fuentes-Afflick E, Hessol NA, Perez-Stable EJ. Testing the epidemiologic paradox of low birth weigth in Latinos. Arch Pedriatr Adolesc Med. 1999; 153:147-53.

42. Silveira MF, Santos IS, Matijasevich A, Malta DC, Duarte EC. Nascimentos pré-termo no Brasil entre 1994 e 2005 conforme o Sistema de Informaçóes sobre Nascidos Vivos (SINASC). Cad Saúde Pública. 2009; 25(6):1267-75.

43. Silveira MF, Santos IS, Barros AJD, Matijasevich A, Barros FC, Victora CG. Aumento da prematuridade no Brasil: revisão de estudos de base populacional. Rev Saúde Pública. 2008; 42:957-64.

44. Silva AAM, Silva LM, Barbieri MA, Bettiol H, Carvalho LM, Ribeiro VS, et al. The epidemiologic paradox of low birth weight in Brazil. Rev Saúde Pública. 2010; 44(5):767-75.

45. Kramer MS. Determinants of low birth weight: methodological assessment and meta-analysis. Bulletin of the World Health Organization. 1987; 65(5):663-737.

46. Silveira DS, Santos IS. Adequação do pré-natal e peso ao nascer: uma revisão sistemática. Cad Saúde Pública 2004; 20(5):1160-8.

47. Barros FC, Barros AJD, Villar J, Matijasevich A, Domingues MR, Victora CG. How many low birthweight babies in low- and middle income countries are preterm? Rev Saúde Pública 2011; 45(3):607-16.

48. Moraes AB, Zanini RR, Giugliane ERJ, Riboldi J. Tendência da proporção de baixo peso ao nascer, no período de 19942004, por microrregião do Rio Grande do Sul, Brasil: uma análise multinível. Cad Saúde Pública. 2011; 27(2):229-40.

49. Sclowitz IKT, Santos IS. Fatores de risco na recorrência do baixo peso ao nascer, restrição de crescimento intrauterino e nascimento pré-termo em sucessivas gestaçóes: um estudo de revisão. Cad Saúde Pública. 2006; 22(6):1129-36.

50. Mello Jorge MHP, Laurenti R, Gotlieb SLD. Análise da qualidade das estatísticas vitais brasileiras: a experiência de implantação do SIM e do SINASC. Ciênc Saúde Coletiva. 2007; 12(3):643-54.

51. Romero DE, Cunha CB. Avaliação da qualidade das variáveis epidemiológicas e demográficas do Sistema de Informaçóes sobre Nascidos Vivos, 2002. Cad Saúde Pública. 2007; 23(3):701-14.

52. Szwarcwald CL. Strategies for improving the monitoring of vital events in Brazil. International Journal of Epidemiology. 2008; 37:738-44.

53. Figueroa Pedraza D. Qualidade do Sistema de Informaçôes sobre Nascidos Vivos (Sinasc): análise crítica da literatura. Ciênc Saúde Colet. 2012; 17(10):2729-37. 